J. Lake Sci.(湖泊科学), 2017, 29(4): 804-810

DOI 10. 18307/2017. 0403

(c) 2017 by Journal of Lake Sciences

\title{
洱海湖滨带冬季水鸟的水质指示作用”
}

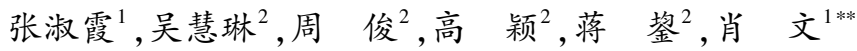 \\ (1: 大理大学东喜玛拉雅研究院,大理 671003) \\ (2: 大理大学农学与生物科学学院,大理 671003)
}

摘 要: 水鸟在物种或群落水平上可以对湿地营养状态有所响应, 预警生态系统有害的改变. 以大型淡水湖泊洱海为例, 基于 2015 年 2 月沿岸 17 个观察点的冬季水鸟调查与水质同步监测, 研究小瓶鹤 (Podiceps ruficollis)、白骨顶 (Fulica atra) 和黑水鸡 (Gallinula chloropus) 密度对沿岸水体中总磷和总氮浓度的响应关系. 结果发现白骨顶和小鹉殷密度均与总氮浓 度呈显著正相关, 也均与总磷浓度呈显著正相关; 黑水鸡密度与总氮和总磷浓度无显著相关性. 结果表明, 水鸟可以在物 种水平上对洱海湖滨带水中营养物质变化有所响应, 也强调了根据具体指示物种的生态学习性选择水鸟指示物种尤为 重要. 白骨顶和小扸鸺在更大空间和时间尺度上的水质指示作用有待于更多湖泊或水库水体的长期相关研究.

关键词: 淡水湖;生物指示;水鸟;水质; 洱海

\section{The bio-indicator role of wintering waterbirds in littoral zone of Lake Erhai, Yunnan Prov- ince}

\author{
ZHANG Shuxia ${ }^{1}$, WU Huilin ${ }^{2}$, ZHOU Jun ${ }^{2}$, GAO Ying ${ }^{2}$, JIANG Jun ${ }^{2} \&$ XIAO Wen ${ }^{1 * *}$ \\ (1: Institute of Eastern-Himalaya Biodiversity Research, Dali University, Dali 671003, P.R.China) \\ (2: Agriculture and Biology College, Dali University, Dali 671003, P.R.China)
}

\begin{abstract}
Waterbirds respond sensitivly to the nutrient change of wetlands in a species-specific or community way, which can provide early warning for changes that could negatively affect ecosystems. We studied the relationship between waterbird density and water quality index among 17 lakeshore sections in Lake Erhai, a large freshwater lake. We counted individual numbers of Little Grebe (Podiceps ruficollis), Common Coot (Fulica atra), Common Moorhen (Gallinula chloropus) and collected water samples synchronously in February 2015. Pearson correlation analysis showed that the population densities of both Common Coot and Little Grebe were significantly correlated to the total nitrogen concentration and the total phosphorus concentration. While the population density of Common Moorhen was not correlated to either the total nitrogen concentration or the total phosphorus concentration. The results suggested that waterbirds could respond to nutrient changes in littoral zone of Lake Erhai in a species-specific way, and a thorough knowledge of the ecology of a given species is required to properly choose a waterbird bio-indicator species. Further studies in more lakes or reservoirs could justify the bio-indicator role of Common Coot and Little Grebe at larger temporal and spatial scales. Keywords: Freshwater lake; bio-indicator; waterbirds; water quality; Lake Erhai
\end{abstract}

水鸟是湿地中较容易监测的生物类群,水鸟的强运动能力使其对湿地中食物资源的变化反应迅速 ${ }^{[1-2]}$. 在水生态系统中,由于不同生物类群对相同环境变量反应的趋同性很弱,因此应仔细挑选特定生物类群,使 其对特定环境变量有所响应 ${ }^{[3-4]}$. 已有的研究表明水鸟能够对湿地水环境变量变化有所响应 ${ }^{[1,5]}$. 水鸟既对 湿地营养状态 ${ }^{[6-7]}$, 也对湿地水文过程有所响应 ${ }^{[8-10]}$. 水鸟群落指示河流河岸带人为干扰和水质状况的研究

* 国家水体污染控制与治理科技重大专项( 2013ZX07105-005-02-02,2012ZX07105-003-01-01)、云南省高校洱海流域 保护与可持续发展研究重点实验室项目、中国三江并流区域生物多样性协同创新中心项目和大理大学博士科研 启动项目联合资助. 2016-04-20 收稿;2016-08-02 收修改稿. 张淑霞 (1977 ), 女, 博士, 高级工程师; E-mail: Zhangsx@ eastern-himalaya.cn.

** 通信作者; E-mail:Xiaow@ eastern-himalaya.cn. 
也得到了广泛的关注 ${ }^{[11-13]}$.

影响水鸟对栖息地利用的因素近半个世纪以来在鸟类学界也得到了广泛的研究,也从另一个角度说明 了水鸟这一生物类群对湿地环境因子的响应, 例如 $\mathrm{Ma}$ 等 ${ }^{[14]}$ 综述认为湿地的水深、水位波动、植被、盐度、食 物资源、食物资源可获得性、湿地大小和湿地连通性均是影响水鸟对栖息地利用的重要因素; 除此之外,湿 地的底泥和水质特征也可以直接或间接的影响水鸟对湿地的利用. 除环境因素外, 人为干扰因素也会影响 水鸟对栖息地的利用 ${ }^{[15]}$.

虽然水鸟作为湿地指示物种早期曾一度被怀疑, 因为它们或许缺乏与湿地中其他生物类群或湖沼学变 量变化的直接可测量响应. 虽然在某些情况下,鸟类并不与以与其他生物类群相同的方式对湿地特征响应, 这时鸟类可能不适宜指示其他监测类群的变化; 然而, 在另一些情况下, 鸟类可以是营养状态, 鱼类资源或 水生植物多度可靠的指示生物 ${ }^{[1]}$. 另一方面, 因为水鸟与湖泊中的非生物环境和食物网具有很密切的关系, 越来越多的研究者开始尝试将水鸟整合进湖泊生态系统的研究中 ${ }^{[16]}$, 而且已有研究也表明水鸟的确对湿地 水体的营养状态或水质指标变化有所响应, 而且是以物种或群落的方式有所响应 ${ }^{[17-19]}$. 在西班牙东南部的

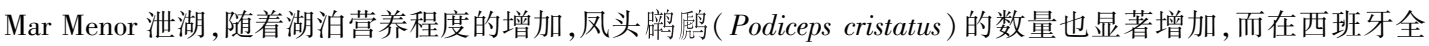

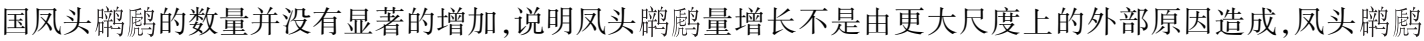
是对湖泊营养状态有所响应的水鸟物种 ${ }^{[20]}$; 在该泄湖富营养化晚期阶段, 白骨顶 (Fulica atra) 则是水质状 态变化的响应物种 ${ }^{[17]}$. 在美国佛罗里达州的大沼泽地, 黑水鸡 (Gallinula chloropus) 在富营养的水域中数量 更多 ${ }^{[21]}$. 综上, 在特定物种对湿地营养状态的响应上, 凤头䴙殷、白骨顶和黑水鸡是对水体营养程度有所响 应的水鸟物种,也说明在物种水平上开展相关研究的重要性.

对 Mar Menor 泄湖局部湖滨带分段进行的水鸟物种与营养盐之间响应关系的研究表明, 特定水鸟物种 的密度并不与沿岸营养盐变化有响应关系 ${ }^{[18]}$. 在大型淡水湖泊, 人类对物种水平上水鸟与不同片区水质的 响应关系知之甚少. 已开展的滇池和洱海水生植物覆盖状况与水质关系的研究表明, 营养盐含量较高的湖 滨带, 水生植物覆盖度较高 ${ }^{[22]}$, 而水鸟依赖水生植物提供的食物来源或庇护场所生存 ${ }^{[23]}$, 因此, 预期营养盐 含量高的湖滨带, 指示水鸟的数量也将较大.

洱海是云南省第二大淡水湖泊, 属国家重点保护的水域之一, 具有农田灌溉、工业、生活用水和水能利 用的功能 ${ }^{[24-25]}$. 近年来, 韩联宪等对洱海西岸湖滨带的陆地鸟类和水鸟多样性进行了研究, 共记录到鸟类 115 种, 离岸 $200 \mathrm{~m}$ 内近岸明水面的优势种为白骨顶、小辟鹰 (Podiceps ruficollis)、黑水鸡、赤麻鸭 (Tadorna ferruginea) 和红嘴鸥 (Larus ridibundus) ${ }^{[26]}$. 本研究以洱海为研究地点, 研究小辟傂、白骨顶和黑水鸡的个体 数量对沿岸水体中 TP 和 TN 浓度的响应关系, 检验上述 3 种水鸟数量是否与沿岸水体中 TN 和 TP 浓度均 具有相关关系, 评价鸟类这一生物类群对淡水湖泊湖滨带水质变化是否具有响应关系; 通过水鸟对水质指 示作用的探讨性研究,为未来开展湖滨带水质的快速生物评价提供基础资料.

\section{1 研究方法}

\section{1 研究区域概况}

洱海 ( $25^{\circ} 36^{\prime} \sim 25^{\circ} 58^{\prime} \mathrm{N}, 100^{\circ} 06^{\prime} \sim 100^{\circ} 18^{\prime} \mathrm{E}$ ) 位于云南省大理白族自治州境内, 是一个典型的内陆断陷 淡水湖泊. 水位 $1973.66 \mathrm{~m}$ 时, 长 $42.58 \mathrm{~km}$, 最大宽 $8.0 \mathrm{~km}$, 湖面面积 $249.0 \mathrm{~km}^{2}$, 最大水深 $20.7 \mathrm{~m}$, 平均水深 $10.17 \mathrm{~m}^{[25]}$. 洱海湖滨带全长 $128 \mathrm{~km}$, 其中西部 $48 \mathrm{~km}$, 南部 $8 \mathrm{~km}$, 东部 $51 \mathrm{~km}$, 北部 $11 \mathrm{~km}^{[27]}$. 湖区属中亚热 带高原季风气候, 年平均气温 $15.0^{\circ} \mathrm{C}, 1$ 月平均气温 $8.5^{\circ} \mathrm{C}$, 极端最低气温 $-4.2^{\circ} \mathrm{C}, 7$ 月平均气温 $20.1^{\circ} \mathrm{C}$, 极端 最高气温 $34.0^{\circ} \mathrm{C}$. 多年平均日照时数 $2472 \mathrm{~h}$, 无霜期 $305 \mathrm{~d}$, 年均降水量 $1056.6 \mathrm{~m}$, 最大年降水量 $1456.5 \mathrm{~mm}$, 最小年降水量 $650.2 \mathrm{~mm}, 5-10$ 月雨季降水量占全年的 $85 \%$ 96\% ${ }^{[25]}$. 近 50 a 来,随着洱海流域社会经济的 快速发展, 进人洱海的氮、磷负荷持续增加, 洱海水质持续下降, 同时水位大幅波动, 从而导致洱海水生态系 统发生退化, 沉水植物多样性下降且群落结构趋于简单化 ${ }^{[28]}$.

\section{2 调查方法}

根据洱海水鸟聚集点的分布,避开密集的休闲娱乐人群,在洱海环湖湖滨带选择了 17 个水鸟观察点, 2 个观察点之间最小距离至少 $1 \mathrm{~km}$, 或有明显的边界标志 (图 1). 2015 年 1 月 25-26 日快速完成一次环湖水 
鸟调查和水样同步采集. 水鸟调查由 2 个调查人员完成, 对每个观察点以观察点为圆心, 半径 $1 \mathrm{~km}$ 内水域 中的小䴙鹛、黑水鸡和白骨顶进行分种计数. 使用莱卡 20-60×77 变焦望远镜 (Leica APO TELEVID 77, 产地 德国) 和 10×42 双筒望远镜 (Olympus EXWP I, 产地日本), 采用 “Look-See” 方法 ${ }^{[29]}$ 对视野内的水鸟进行观 察. 调查日均为晴天, 且风浪小, 调查仅在有日照时间段内进行 $(09: 00-17: 00)$, 调查时段内的观察点水域 均无船只活动.

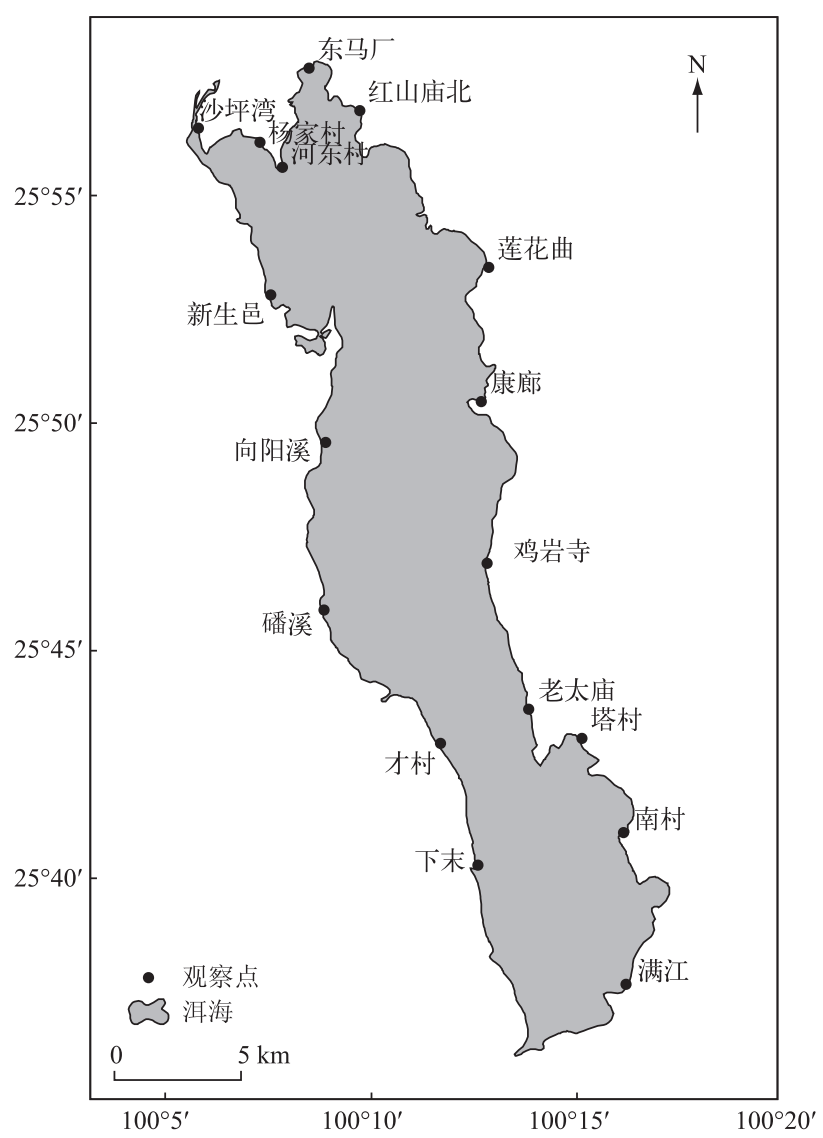

图 1 洱海湖滨带冬季水鸟观察点位

Fig. 1 The wintering waterbirds observation sites in lakeshore of Lake Erhai

水鸟观察计数的同时, 在每个水鸟观察点近岸区域 (离岸 $0 \sim 60 \mathrm{~m}$ 范围), 由第 3 个调查人员专门采集上 覆水样, 全湖共采集 17 份. 水样带回实验室后立即进行测定, 总氮( TN) 浓度采用碱性过硫酸钾消解紫外分 光光度法测定; 总磷 $(\mathrm{TP})$ 浓度采用钿锑抗分光光度法测定 ${ }^{[30]}$.

\section{3 数据分析}

在 ArcGIS 下, 根据每个观察点的 GPS 点位信息计算每个点半径 $1 \mathrm{~km}$ 的缓冲区. 然后, 叠加洱海水域, 获得观察范围内的水域范围. 最后, 根据视野范围内的遮挡 (树林、建筑等), 去除不可观察的范围, 得到每个 点的可观测范围, 并计算观测范围的面积. 根据每个观察点观察到的水鸟数量和实际观察水面面积分种计 算水鸟的密度. 在 SPSS 13.0 软件中完成小辟挀鸟、黑水鸡和白骨顶密度 (只 $/ \mathrm{hm}^{2}$ ) 和 TN 、TP 变量的描述性统 计, 使用 Kolmogorov-Smirnov test 检验数据的分布是否符合正态分布, 对符合正态分布的变量采用 Pearson 相 关性分析. 


\section{2 结果}

\section{1 洱海湖滨带常见水乌与水质的变异}

洱海湖滨带中白骨顶、黑水鸡和小辟虛 3 种常见水鸟密度和水质指标变异均较大 (表 1). 白骨顶密度最大, 平均值为 0.975 只 $/ \mathrm{hm}^{2}$; 密度最大值为 5.316 只 $/ \mathrm{hm}^{2}$, 出现在东南岸 的塔村观察点; 最小值为 0 , 出现在东岸鸡岩 寺、康廊、莲花曲等多个观察点. 黑水鸡密度 最小, 平均值为 0.07 只 $/ \mathrm{hm}^{2}$, 密度最大值为 0.322 只 $/ \mathrm{hm}^{2}$, 出现在北岸的东马厂观察点; 最小值为 0 ,出现在东岸鸡岩寺、康廊、莲花曲 和北岸河东村、杨家村等多个观察. 小鹉愿的密度最大值为 1.779 只 $/ \mathrm{hm}^{2}$, 出现在西北岸的沙坪湾; 最小值 为 0 ,出现在东岸老太庙观察点.

在水质指标中, $\mathrm{TP}$ 浓度平均值为 $0.086 \mathrm{mg} / \mathrm{L}$, 处于 $\mathrm{IV}$ 类水水平; $\mathrm{TN}$ 浓度平均值为 $0.709 \mathrm{mg} / \mathrm{L}$, 处于 III 类 水水平. TP 和 TN 浓度的最大值分别为 0.313 和 $1.732 \mathrm{mg} / \mathrm{L}$, 均出现在洱海西北岸的沙坪湾观察点, TP 浓度 的最小值为 $0.049 \mathrm{mg} / \mathrm{L}$, 出现在东南岸的南村观察点; TN 浓度的最小值为 $0.293 \mathrm{mg} / \mathrm{L}$, 出现在东岸的老太庙 观察点. 根据水质监测结果, 沙坪湾观察点的水质已达劣 $\mathrm{V}$ 类, 部分观察点的水质为 $\mathrm{V}$ 类, 包括西岸的才村 $(\mathrm{TP}=0.105 \mathrm{mg} / \mathrm{L})$ 、新生邑 $(\mathrm{TN}=1.694 \mathrm{mg} / \mathrm{L})$ 和东岸的康廊 $(\mathrm{TN}=0.117 \mathrm{mg} / \mathrm{L})$ 观察点.

\section{2 水鸟密度与水质的相关性}

经过 Kolmogorov-Smirnov test 检验, 认为 各变量数据基本均符合正态分布. 对 5 个变 量两两之间进行 Pearson 相关分析 (表 2) , 发 现白骨顶密度 $(r=0.613, P=0.009)$ 、小䴙 㕐密度 $(r=0.550, P=0.022)$ 均与 $\mathrm{TN}$ 浓度 呈显著正相关; 白骨顶密度 $(r=0.511, P=$ $0.036)$ 、小啯胍鸟密度 $(r=0.532, P=0.028)$ 均与 TP 浓度呈显著正相关. 白骨顶密度与 小脱鹤的密度分布呈显著正相关 $(r=$ $0.532, P=0.028), \mathrm{TP}$ 与 $\mathrm{TN}$ 浓度呈显著正 相关 $(r=0.603, P=0.010)$.
表 1 洱海湖滨带 17 个观察点 3 种水鸟密度和 水质指标的描述性统计

Tab.1 Summary statistics for three waterbirds densities and water quality index in 17 shoreline sections of Lake Erhai

\begin{tabular}{lcccc}
\hline 变量 & 平均值 & 最小值 & 最大值 & 标准误 \\
\hline 白骨顶 $/\left(\right.$ 只 $\left./ \mathrm{hm}^{2}\right)$ & 0.975 & 0 & 5.316 & 0.413 \\
黑水鸡 $/\left(\right.$ 只 $\left./ \mathrm{hm}^{2}\right)$ & 0.070 & 0 & 0.322 & 0.026 \\
小辟鹤 $/\left(\right.$ 只 $\left./ \mathrm{hm}^{2}\right)$ & 0.561 & 0 & 1.779 & 0.108 \\
$\mathrm{TP} /(\mathrm{mg} / \mathrm{L})$ & 0.086 & 0.049 & 0.313 & 0.015 \\
$\mathrm{TN} /(\mathrm{mg} / \mathrm{L})$ & 0.709 & 0.293 & 1.732 & 0.101 \\
\hline
\end{tabular}

表 2 洱海湖滨带 17 个观察点 3 种水鸟密度与 水质指标的 Pearson 相关分析结果

Tab.2 Pearson correlation matrix for three waterbirds densities and water quality index in 17 shoreline sections of Lake Erhai

白骨顶密度 黑水鸡密度 小鸲鹤密度 TP TN
白骨顶密度
黑水鸡密度
小䴙鹤密度
0.366
0.532 *
0.110
$\mathrm{TP}$
0.511 *
0.291
$0.666^{*}$
$0.613^{*} \quad 0.245$
$0.550 * 0.603 *$

* 表示在 0.05 水平 (双侧) 上显著相关.

\section{3 讨论}

\section{1 指示水质的水鸟物种选择}

指示水质变化的水鸟应严格依赖水体受食活动, 并且容易被观察到 ${ }^{[14]}$. 虽然冬季洱海也有相当数量的 雁鸭类 ${ }^{[26]}$,但是雁鸭类性机警,对人类干扰活动较敏感,一般在距岸边观察点接近或超过 $1 \mathrm{~km}$ 的水域中活 动,雁鸭类作为洱海的近岸水质指示并不合适; 另外,部分雁鸭类可能仅选择洱海作为日间休息地,不进行 受食活动, 因此综合多种因素, 雁鸭类不适于作为洱海近岸水质的指示生物. 对于路类等涉禽来说, 其受食 地并不严格依赖水体,有时在附近农田中受食, 因此也不符合水质指示物种的要求. 洱海冬季虽然为较多红 嘴鸥利用, 但是红嘴鸥的食物来源部分来自湖泊外部, 例如人类的投喂食物, 因此鸥类也未被选作水质指示

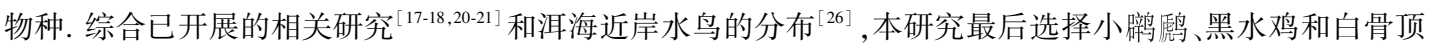
3 种水鸟尝试研究其水质指示作用,这 3 种水鸟严格依赖洱海近岸水体及其底质受食.

\section{2 观察点水域水质的代表性}

洱海在弱风浪水动力扰动下, 湖滨带近岸 (离岸 $0 \sim 60 \mathrm{~m}$ 范围) 区域水体 TN 、TP 浓度与远岸 (离岸 70 
$190 \mathrm{~m}$ 内) 差异不显著 ${ }^{[27]}$; 在西班牙泄湖 Mar Menor, 在离岸 $1 \mathrm{~km}$ 以内, 营养盐浓度随着距岸边距离增加而 快速下降, 不同湖滨带的水质差异在离岸 $50 \mathrm{~m}$ 内就得以充分体现 ${ }^{[17]}$. 本研究的重点在于区分洱海内不同湖 滨带之间的水质和水鸟差异, 每个采样点都在离岸 $50 \mathrm{~m}$ 以内的地方采用相同的方案采集水样, 已经反映了 洱海不同湖滨带之间的水质差异; 17 个观察点 TN 和 TP 浓度均呈现较大的变异, TN 浓度在 $0.293 \sim 1.732$ $\mathrm{mg} / \mathrm{L}$ 之间, TP 浓度在 0.049 0.313 $\mathrm{mg} / \mathrm{L}$ 之间, 且与已开展的洱海湖滨带水质研究结果 ${ }^{[27]}$ 相似, 即在空间 上呈现北部营养盐浓度最高, 东部营养盐浓度最低的格局; 造成湖滨带水质发生空间变异的原因为不同湖 滨带周边的土地利用、沿岸生产方式有所差异, 从而可能导致相应湖滨带的人湖营养盐总量有较大差异. 在 今后的深人研究中, 在水鸟观察范围内按距离分层取水样将更为精细地揭示水鸟对湖滨带水质的响应规律.

\section{3 洱海湖滨带 3 种水鸟对水质变化的响应}

在洱海整个湖滨带水体水质空间变异大的背景下, 本研究中白骨顶和小鹉髟的种群密度均与 TN 和 TP 浓度呈显著正相关, 即随着湖滨带不同观察点水体中 TN 和 TP 浓度的增加, 白骨顶和小㸟鹤的数量亦增加. 研究结果不同于已开展的物种水平上研究,西班牙泄湖 Mar Menor 西端长约 $4.12 \mathrm{~km}$ 的湖滨带, 白骨顶和辟鸟 鹧类水鸟密度对湖滨带营养盐的变化并没有响应, 水鸟对沿岸淡水资源的依赖可能是其没有响应的原

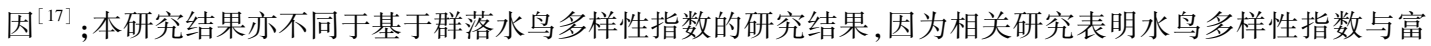
营养指数、污染程度呈负相关关系 ${ }^{[31-32]}$, 而与北美佛罗里达州基于 46 个湖泊的群落研究结果相似, 表明水 鸟群落物种丰富度和多度与 TN 和 TP 浓度均呈正相关 ${ }^{[6]}$. 采用不同的水鸟响应变量以及差别较大的研究地 环境条件可能是造成研究结果不同的主要原因.

在其他生物类群中, 对洱海浮游植物群落多度影响最大的水环境因子为 TN 和 TP 浓度 ${ }^{[33]}$, 影响洱海轮 虫分布的环境因子包括 $\mathrm{TN}$ 浓度 ${ }^{[34]}$; 对洱海底栖动物群落变异的水环境因子为 $\mathrm{TP}$ 和 $\mathrm{Ca}^{2+}$ 浓度 ${ }^{[35]}$. 综上, 本 研究和已开展的研究表明水鸟同湖泊中其他生物类群一样, 可以对洱海湖滨带水体营养盐 TN 和 TP 浓度变 化表现出响应关系.

与美国佛罗里达州 everglades 湿地相关研究 ${ }^{[21]}$ 不同的是, 黑水鸡并没有对洱海湖滨带水质有所响应, 这 可能与黑水鸡在洱海的生境偏好有关. 根据观察, 黑水鸡与白骨顶和小䳄鸺的生境偏好并不相同, 虽然黑水 鸡也在靠近岸边的区域活动, 但多数时间是在紧邻岸边的挺水植物和浮水植物从中活动, 而白骨顶和小䃅 扸通常是在距离岸边有一定距离的明水面活动. 本研究的水质采样点并不在挺水植物和浮水植物带内, 这 可能是黑水鸡未对洱海湖滨带水质有所响应的原因, 也说明了在物种水平上根据物种的具体生态学习性来 选择水鸟指示物种尤为重要 ${ }^{[1]}$.

\section{4 结论}

鸟类是开展生物多样性监测的重要指示类群, 未来我国将大力发展全国范围内的鸟类监测工作 ${ }^{[36]}$. 水 鸟作为湿地水质生物指示虽有一定局限性 ${ }^{[1]}$, 但本研究认为白骨顶和小铇瓜鸟是对洱海湖滨带近岸水体 TN、 $\mathrm{TP}$ 浓度有一定响应关系的物种, 在物种水平上开展相关监测工作可能是提高水鸟水质指示作用的途径. 在 未来冬季洱海湖滨带水质快速评价时, 可以尝试使用这 2 个物种作为洱海冬季近岸水体水质状态的指示生 物. 白骨顶和小憎鹤在更大空间和时间尺度上的水质指示作用有待于更多湖泊或水库水体的长期相关 研究.

\section{5 参考文献}

[ 1 ] Amat JA, Green AJ. Waterbirds as bioindicators of environmental conditions. In: Hurford C, Schneider M, Cowx I eds. Conservation monitoring in freshwater habitats: A practical guide and case studies. Netherlands: Springer, 2009: 45-52.

[ 2 ] Zhang Shuxia, Dong Yunxian, Xia Feng. Significance of waterbird monitoring in lake ecosysytems. J Lake Sci, 2011, 23 (2) : 155-162. DOI: 10.18307/2011.0201. [张淑霞, 董云仙, 夏峰. 湖泊生态系统的水鸟监测意义. 湖泊科学, $2011, \mathbf{2 3}(2)$ : 155-162. ]

[ 3 ] Heino J. Are indicator groups and cross-taxon congruence useful for predicting biodiversity in aquatic ecosystems. Ecological Indicators, 2010, 10: 112-117.

[ 4 ] Guareschi S, Abellán P, Laini A et al. Cross-taxon congruence in wetlands: Assessing the value of waterbirds as surrogates 
of macroinvertebrate biodiversity in Mediterranean Ramsar sites. Ecological Indicators, 2014, 49: 204-215.

[ 5 ] Cintra R. Spatial distribution and composition of waterbirds in relation to limnological conditions in the Amazon basin. Hydrobiologia, 2015, 747: 235-252.

[ 6 ] Hoyer MV, Canfield DE. Bird abundance and species richness on Florida lakes: Influence of trophic status, lake morphology, and aquatic macrophytes. Hydrobiologia, 1994, 297/280: 107-119.

[ 7 ] Fernández JM, Selma MAE, Aymerich FR et al. Aquatic birds as bioindicators of trophic changes and ecosystem deterioration in the Mar Menor lagoon (SE Spain). Hydrobiologia, 2005, 550: 221-235.

[ 8 ] Paillisson JM, Reeber S, Marion L. Bird assemblages as bio-indicators of water regime management and hunting disturbance in natural wet grasslands. Biological Conservation, 2002, 106: 115-127.

[ 9 ] Chen Bing, Cui Peng, Liu Guanhua et al. Relationships between changing water levels and numbers of wintering tuber-eating birds in Poyang Lake National Nature Reserve. J Lake Sci, 2014, 26(2) : 243-252. DOI:10.18307/2014.0211. [陈 冰, 崔鹏, 刘观华等. 鄱阳湖国家级自然保护区食块茎鸟类种群数量与水位的关系. 湖泊科学, 2014, 26 (2) : 243-252.]

[10] Hu Zhenpeng, Ge Gang, Liu Chenglin. Response of wintering migratory birds to hydrological processes in Poyang Lake. Journal of Natural Resources, 2014, 29(10): 1770-1779. [ 胡振鹏, 葛刚, 刘成林. 越冬候鸟对鄱阳湖水文过程的响 应. 自然资源学报, 2014, 29(10): 1770-1779.]

[11] Bryce SA, Hughes RM, Kaufmann PR. Development of a bird integrity index: Using bird assemblages as indicators of riparian condition. Environmental Management, 2002, 30(2) : 294-310.

[12] Bryce SA. Development of a bird integrity index: Measuring avian response to disturbance in the Blue Mountains of Oregon, USA. Environmental Management, 2006, 38(3) : 470-486.

[13] Larsen S, Sorace A, Mancini L. Riparian bird communities as indicators of human impacts along Mediterranean streams. Environmental Management, 2010, 45(2): 261-273.

[14] Ma Z, Cai Y, Li B et al. Managing wetlands habitats for waterbirds: An international perspective. Wetlands, 2010,30 (1) : 15-27.

[15] Tavares DC, Guadagnin DL, Moura JF et al. Environmental and anthropogenic factors structuring waterbird habitats of tropical coastal lagoons: Implications for management. Biological Conservation, 2015, 186: 12-21.

[16] Kerekes JJ, Pollards JB. Aquatic birds in the trophic web of lakes. Developments inhydrobiology (Vol.96). Netherlands: Springer, 1994.

[17] Robledano F, Esteve MA, Martínez-Fernández P et al. Determinants of wintering waterbird changes in a Mediterranean coastal lagoon affected by eutrophication. Ecological Indicators, 2011, 11: 395-406.

[18] Robledano F, Pagan I, Francisco J. Waterbirds and nutrient enrichment in Mar Menor Lagoon, a shallow coastal lake in southeast Spain. Lake \& Reservoirs: Research and Management, 2008, 13: 37-49.

[19] Nilsson SG, Nilsson IN. Breeding bird community densities and species richness in lakes.Oikos, 1978, 31: 214-221.

[20] Fernández JM, Selma M, Aymerich FR et al. Aquatic birds as bioindicators of trophic changes and ecosystem deterioration in the Mar Menor lagoon (SE Spain). Hydrobiologia, 2005, 550(1) : 221-235.

[21] Crozier GE, Gawlik DE. Avian response to nutrient enrichment in an oligotrophic wetland, the Florida everglades. The Condor, 2002, 104: 631-642.

[22] Xie Jie, Wu Deyi, Chen Xuechu et al. Relationship between aquatic vegetation and water quality in littoral zones of Lake Dianchi and Lake Erhai. Environmental Sciences \& Technology, 2013, 36(2) : 55-59. [谢杰, 吴德意, 陈雪初等. 滇池 和洱海湖滨带水生植被状况与水质的关系研究. 环境科学与技术, 2013, 36(2): 55-59.]

[23] Ma ZJ, Cai YT, Li B et al. Managing wetland habitats for waterbirds: An international perspective. Wetlands, 2010,30 : $15-27$.

[24] Wei Zhihong, Yang Zhenxiang, Tang Xiongfei et al. Three-Dimensional Modeling study of water quality processes and characteristics in Erhai Lake. Journal of Kunming University of Science and Technology (Nature Science Edition), 2013, 28(2) : 93-101. [ 卫志宏, 杨振祥, 唐雄飞等. 洱海湖泊及湖湾水质水生态模型及特征分析. 昆明理工大学学报 (自然科学版), 2013, 28(2): 93-101.]

[25] Wang Sumin, Dou Hongshen eds. Chinese lakes. Beijing: Science Press, 1998: 371. [王苏民, 窦鸿身. 中国湖泊志. 北 京: 科学出版社, 1998: 371.] 
[26] Han Lianxian, Deng Zhangwen, Yan Dao. Bird diversity in shore habitats of Erhai Lake, Yunnan Province. Chinese Journal of Zoology, 2014, 49(2) : 185-194. [ 韩联宪, 邓章文, 岩道. 大理洱海湖滨带鸟类多样性. 动物学杂志, 2014, 49(2) : 185-194.]

[27] Yin Yanzhen, Chu Zhaosheng, Zhao Ming et al. Spatial and temporal changes in water quality in water quality in aquaticterrestrial ecotone of Lake Erhai. China Environmental Science, 2011, 31(7): 1192-1196. [尹延震, 储昭升, 赵明等. 洱海湖滨带水质的时空变化规律. 中国环境科学, 2011, 31(7) : 1192-1196.]

[28] Fu Hui, Yuan Guixiang, Cao Te et al. Succession of submerged macrophyte communities in relation to environmental change in Lake Erhai over the past 50 years. J Lake Sci, 2013, 25(6) : 854-861. DOI: 10.18307/2013.0609. [符辉, 袁 桂香, 曹特等. 洱海近 $50 \mathrm{a}$ 来沉水植被演替及其主要驱动因素. 湖泊科学, 2013, 25(6) : 854-861.]

[29] Bibby CJ, Burgess ND, Hill DA et al. Bird Census Technique. 2nd ed. Lodon: Academic Press, 2000: 161.

[30] Editorial Board of Water and Wastewater Monitoring and Analysis Methods, Ministry of Environmental Protection of the People's Republic of China eds. Water and wastewater monitoring and analysis methods: 4th edition. Beijing: China Environmental Science Press, 2002. [国家环境保护总局《水和废水监测分析方法》编委会. 水和废水监测分析方法:第 4 版. 北京: 中国环境科学出版社, 2002.]

[31] He Xiaofang, Wu Faqing, Zhou Qiaohong et al. Research on waterbirds community feature and its relationship with the eutrophication in Chenhu wetland. Resources and Environment in the Yangtze Basin, 2015, 24(9): 1499-1506. [何小芳, 吴 法清, 周巧红等. 武汉沉湖湿地水鸟群落特征及其与富营养化关系研究. 长江流域资源与环境, 2015, 24(9): 1499-1506. ]

[32] Luo Sha, Hu Hongxing, Cheng Shuiping et al. A primary study on water environment at Lake Jinyinhu, Wuhan. Resources and Environment in the Yangtze Basin, 2010, 19(6):671-677. [罗莎, 胡鸿兴, 成水平等. 武汉市金银湖水鸟多样性 及其与水环境关系的初步研究. 长江流域资源与环境, 2010, 19(6) :671-677.]

[33] Wang Xianli, Jiang Guoqiang, Zhou Wen et al. TMDL of Lake Erhai based on water ecological features. J Lake Sci, 2016, 28(2) : 271-280. DOI: 10.18307/2016.0205. [王显丽, 姜国强, 周雯等. 基于洱海水生态特征的流域最大日负荷总 量控制. 湖泊科学, 2016, 28(2): 271-280.]

[34] Dong Yunxian. Relationship of Rotifers and ecological environment in Erhai Lake. Environmental Sciences in Yunnan, 2003, 22 (supplement) : 106-108. [董云仙. 洱海轮虫及其与生态环境的关系. 云南环境科学, 2003, 22(增刊)： 106-108. ]

[35] Zhang Min, Cai Qinghua, Tang Tao et al. Macrobenthos community structure and its spatial distribution in Erhai watershed lakes. Chinese Journal of Ecology, 2011, 30(8): 1696-1702. [张敏, 蔡庆华, 唐涛等. 洱海流域湖泊大型底栖动物群 落结构及空间分布. 生态学杂志, 2011, 30(8): 1696-1702.]

[36] Cui Peng, Xu Haigen, Ding Hui et al. Status quo, problems and counter measures of bird monitoring in China. Journal of Ecology and Rural Environment, 2013, 29(3): 403-408. [崔鹏, 徐海根, 丁晖等. 我国鸟类监测的现状、问题与对策. 生态与农村环境学报, 2013, 29(3): 403-408.] 